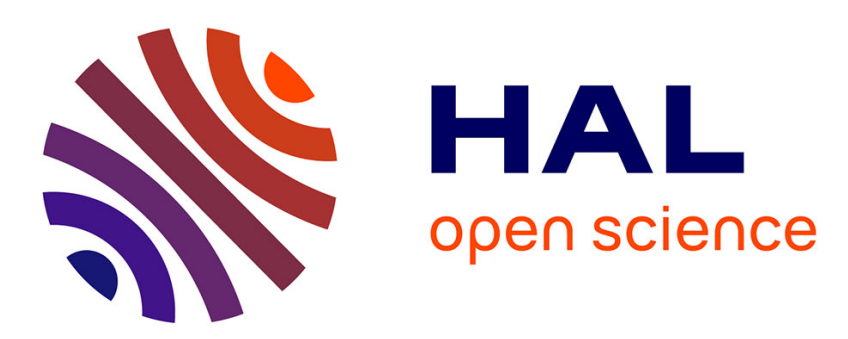

\title{
An experimental approach to prove the efficiency of a cyclic topology of a sensor network
}

Abdellatif Chafik, Francis Lepage

\section{To cite this version:}

Abdellatif Chafik, Francis Lepage. An experimental approach to prove the efficiency of a cyclic topology of a sensor network. International Conference on Complex Systems, ICCS'12, Nov 2012, Agadir, Morocco. pp.CDROM, 10.1109/ICoCS.2012.6458560 . hal-00787419

\section{HAL Id: hal-00787419 \\ https://hal.science/hal-00787419}

Submitted on 12 Feb 2013

HAL is a multi-disciplinary open access archive for the deposit and dissemination of scientific research documents, whether they are published or not. The documents may come from teaching and research institutions in France or abroad, or from public or private research centers.
L'archive ouverte pluridisciplinaire HAL, est destinée au dépôt et à la diffusion de documents scientifiques de niveau recherche, publiés ou non, émanant des établissements d'enseignement et de recherche français ou étrangers, des laboratoires publics ou privés. 


\section{An experimental approach to prove the efficiency of a cyclic topology of a sensor network}

\author{
Abdellatif CHAFIK* \\ Research Centre for Automatic Control of Nancy (CRAN) \\ UMR 7039 Nancy-Universit, CNRS \\ Campus sciences BP 70239 - 54516 Vandœuvre Cedex \\ France \\ Abdellatif.chafik@cran.uhp-nancy.fr
}

\author{
Francis LEPAGE \\ Research Centre for Automatic Control of Nancy (CRAN) \\ UMR 7039 Nancy-Universit, CNRS \\ Campus sciences BP 70239 - 54516 Vandœuvre Cedex \\ France \\ francis.lepage@cran.uhp-nancy.fr
}

\begin{abstract}
The main contribution of this paper is to find an optimal topology to provide radio coverage of the total trajectory of mobile sensor. We propose also a geographic routing algorithm to minimize the transmission time of messages. Then, we show that by using this algorithm we will have a better network performance (energy consumption, transmission delay and packet delivery rate). The geographic routing algorithm proposed is based on the calculation of the position, such that each sensor recognizes the adjacent sensors, its pre-successor and immediate successor geographical positions. Each sensor measures the acceleration nodes and updates its coordinates $x, y$ which is estimated using a Luenberger observer. When a mobile sensor wants to send a message (or relay a message) it compares, through his knowledge of its position, the best path (the closest geographically) between him and the destination and finally transmits the message to the next node in the way. This work was realized and validated by measurements performed on an experimental platform that will be also presented in this paper.
\end{abstract}

Index Terms-sensor network, mobility, topology, location, network performance, observator.

\section{INTRODUCTION}

The applications of mobile sensor networks (Wireless sensor networks - WSN) are various; we can give as example the control and/or supervision of an industrial production line... The mobility reveals new problems relative to fixed sensor networks, such as changing the topology, routing and radio propagation conditions depending on the position. Therefore, designed solutions for fixed nodes cannot be used directly in a mobile environment; it requires high energy consumption and creates a poor quality of service [1], [2].

The WSN are composed of a large number of these small communicating devices (several thousand), deployed in order to explore the area. Each sensor is able to perform three main complementary tasks: measuring a physical value, treatment of these measures, and communicates it over the air.

The sensors by their nature are limited in terms of bandwidth, computing power, available memory and finally power onboard. The deployment is mostly random and can be performed in hazardous areas, which prevent any kind of care and maintenance (for example the battery replacement).

In a geographic routing protocol, a node is supposed to know its geographical position, its neighbors and the destination node. Knowledge of the neighborhood is updated periodically with messages exchanged between nodes. In mobility, the frequency of updating the neighbors table of a sensor is proportional to its speed and the type of application. But it is costly in energy, and reduces the lifetime of the sensors, by consequently the lifetime of the network.

Generally, in sensor networks, the localization has two goals, the first one for the application and another one for the functional communication. At the application level, data collected by the sensor have no interest if the position of the measurement has mentioned in this measure. For example, in the monitoring of temperature in a building, it is possible to know the exact position of each node. But, if the sensor network is used for the temperature monitoring in a forest, the nodes can be deployed from an aircraft for example, and the exact position of most of the sensors may be unknown. Several WSN localization techniques are used to estimate the locations of sensors. In such a network the initial positions of the sensors are unknown. Using information available in specific positions of some sensors in the network, the rest of the sensors can deduct their positions by calculating their distance from the first sensors identified. This is feasible based on a technique such as the time difference of arrival (TOA), angle of arrival and connectivity. We will focus in this paper on a geographic routing, by calculating a position with an accelerometer.

In this case of mobile nodes, describing cyclic trajectories sensors position is a dynamic position occupied by the mobile nodes along their cyclic paths. Relative to the static case, it is only to estimate a fixed position, does not vary with time. For consistent estimation, it is necessary that the path is equipped with full radio coverage. 
The rest of the paper is organized as follows: Section 2 gives an overview of related work and outlining our goals. Section 3 defines the network model. Section 4 describes our platform and develops our approach. Section 5 evaluates the performance of the algorithm proposed. Conclusion and future Works are presented in Section 6.

\section{RELATED WORKS}

A geographic routing is based on location nodes. The efficiency of an algorithm for locating and routing is measured by the satisfaction of the constraints; for example the energy consumption and the choice of the optimal path routing messages to a destination.

\section{A. Geographic routing}

Several studies dealing with geographic routing algorithms [3]-[9]. The geographic routing algorithms presented in the state of art switch between greedy mode and recovery mode, according to the network topology. In a greedy approach (greedy geographic forwarding), the nearest node is selected from the neighboring nodes, it is possible to use the Euclidean distance between the current node and the destination [5].

On the other hand MFR (Most Forward Within Radius) [10] prefers the neighbor with the shortest projected distance on the straight line joining the current node and the destination.

\section{B. Location}

Many methods assume that some sensors (called anchors) know their position via GPS, or human intervention.... The systems location deduced the positions of other nodes based on the coordinates of the anchors sensors. An examples of these methods are described in [2], [11]-[13].

Other techniques allow calculating the distances separate the nodes with their neighbors. The most popular methods that calculate the distance between two neighbors are, the RSSI [14], TOA [15] and AOA [16]. The method RSSI (Received Signal Strength Indicator) measures the signal strength at the receiver, based on theoretical and empirical models, the weakening of the signal received by the receiver will be translated into distance. To refine the measure, we consider that a RSSI value can always be obtained through several consecutive measurements of received power, a mainly in the mobility.

To conclude this section, the geographic routing is well adapted to the sensor networks. In fact, it requires only local information network, in order to give the adapted coverage to the network. The confidence range of the result depends essentially on the environment of network deployment (Indoor, Outdoor).

\section{THE TOPOLOGIE}

\section{A. Problematic}

Illustrated for the proposal of this paper, we consider the following scenario: mobile sensors move by taking a defined trajectory, and they have to send measurements to a fixed sink in order to treat or register them. The sink serves as a gateway to another network on which measurements are transmitted. It is assumed that the antennas are unidirectional and denote the radius $R C$ communications, Two cases are presented:

1) If the distance $D$ between the mobile sensor and the sink is less than $R C$ at any point of the trajectory, the transmission can always be direct between the sensor and the sink.

2) Otherwise, the mobile sensor is sometimes outside the coverage area of the sink as shown in figure1.

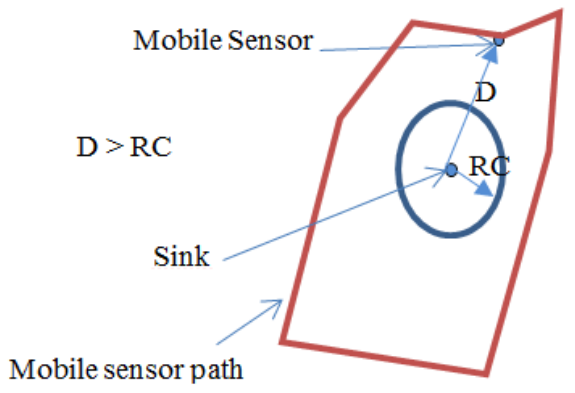

Fig. 1. Example of trajectory with $\mathrm{D}>\mathrm{RC}$

It is necessary to predict fixed and mobile relay nodes to ensure connectivity between the mobile and the sink. The question is about the number of mobile relay in our study. Relay nodes that will ensure communication between a mobile node and the sink must take the same trajectory than the mobile node.

In our case study, and to simplify the problem, we are limited to a circular path and we considered a single mobile node that performs the action. The sink is located close to the path of this node, relay nodes are mobile, they move with the same speed as the mobile node.

\section{B. Topology for circular path}

The purpose of this section is to calculate the minimum number of mobile node " $N$ " must be implanted in the circular path to ensure full coverage of the trajectory of the mobile.

The figure2 shows the study case chosen, in which the mobile sends information to the relay node that is upstream or downstream according to its position in the path.

We consider the vector $\vec{U}$ connecting two closest mobile relay $R_{3}$ and $R_{4}, \vec{W}$ the vector that gives the position of the mobile relay $R_{4}$ relative to the center of the path, and $\vec{V}$ the vector which gives the position of the mobile relay $R_{3} . \beta$ is the angle between the two vectors. Express the angle $\beta$ as a function of communication radius " $R C$ ", the path radius " $r$ " of the mobile, and " $d$ " the Euclidean distance between $R_{3}$ and $R_{4}$.

we have:

$$
\begin{gathered}
\vec{U}=\vec{W}+\vec{V} \\
\|\vec{U}\|^{2}=\|\vec{V}\|^{2}+\|\vec{W}\|^{2}-2\|\vec{V}\| \times\|\vec{W}\| \times \cos (\widehat{\vec{V}, \vec{W}}) \\
\|\vec{V}\|=\|\vec{W}\|=r \\
\|\vec{U}\|=d
\end{gathered}
$$




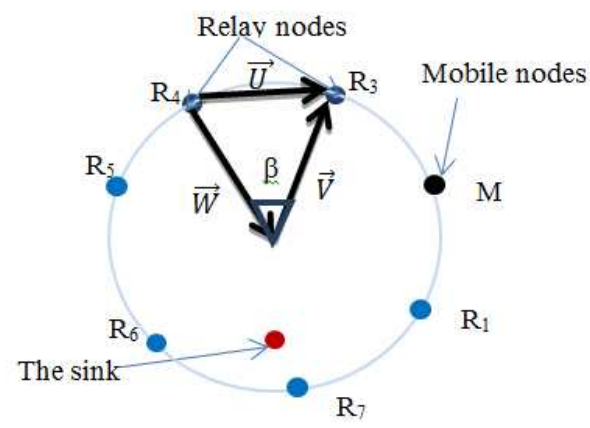

Fig. 2. Circular path for the case $\mathrm{N}=7$

$$
(\widehat{\vec{V}, \vec{W}})=\beta
$$

Expression (2) will be:

$$
\begin{gathered}
d^{2}=2 r^{2}-2 r^{2} \cos (\beta) \\
\beta=\arccos \left(1-\frac{d^{2}}{2 r^{2}}\right)
\end{gathered}
$$

The distance between two neighboring nodes " $d$ " must be less than or equal to the radius of communication $R C \max$, beyond this distance, the nodes cannot communicate properly figure 3.

On the other hand if $d$ is less than $\frac{R C \max }{2}$ the message sent by the sensor at position $i$ is received both by the sensor at position $i+1$ and $i+2$ figure 4 . The value of $N$ should be

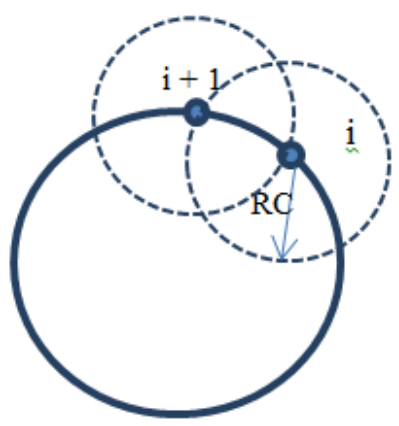

Fig. 3. Case $R C_{\max }$

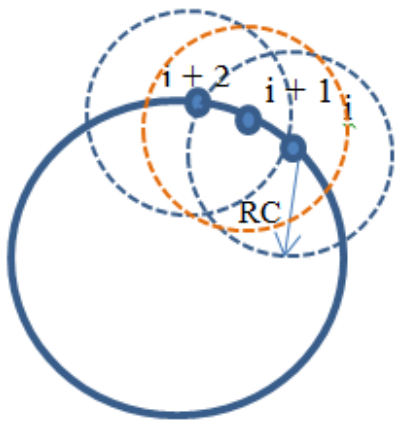

Fig. 4. Case $\frac{R C_{\max }}{2}$ chosen so that " $d "$ is between $R C \max$ and $\frac{R C \max }{2}$.

$$
\left\lceil\frac{2 \pi}{\beta}\right\rceil \leq N<\left\lceil\frac{4 \pi}{\beta}\right\rceil
$$

\section{GEOGRAPHIC ROUTING}

Before deployment, each sensor has a vision of the local network, through the knowledge of its neighbors. The main idea of the geographical routing algorithm is explained as follows: when the mobile node decides to send a message, it must calculate, through knowledge of its position, the best path to the sink. It will transmit this information to the next node in the path. The relay nodes do the same upon receipt of a message. There is another method of communicating periodically the number of hops from the sink period must be very large compared to its speed. The receiver of these messages stocks information in a table that associates each neighbor to the number of hops. This receiver communicated its neighbors. This method is expensive in the energy and rating bandwidth. The position of the mobile is calculated

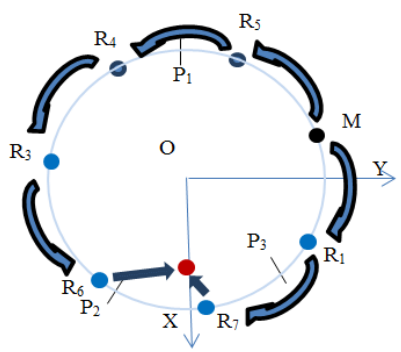

Fig. 5. the possible paths

locally using an acceleration sensor figure6

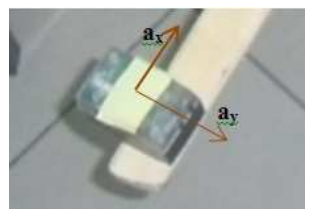

Fig. 6. Mobile node

\section{A. Dynamics of system}

To give a model for this problem, we represent the dynamics of the system by a state model as follow:

$$
\left\{\begin{array}{l}
\dot{X}=A X \\
h(x)=C X
\end{array}\right.
$$

It is assumed that the mobile node has a circular rotation with any speed: $\omega(t)=\dot{\theta}(t)$ We consider in the plane a landmark $(O, X, Y)$ where the origin $O$ is a fixed point, which is the center of the trajectory (figure7). In this case, the position vector is written as:

$$
\left\{\begin{array}{l}
x=R \cos (\theta(t)) \\
y=R \sin (\theta(t))
\end{array}\right.
$$

We deduce the acceleration vector of (10):

$$
\left\{\begin{array}{l}
a_{x}=\ddot{x}=-R \ddot{\theta}(t) \sin (\theta(t))-R(\dot{\theta}(t))^{2} \cos (\theta(t)) \\
a_{y}=\ddot{y}=+R \ddot{\theta}(t) \cos (\theta(t))-R(\dot{\theta})(t)^{2} \sin (\theta(t))
\end{array}\right.
$$

We suppose:

$$
\dot{X}=\left(\begin{array}{c}
\dot{x}_{1} \\
\dot{x}_{2} \\
\dot{x}_{3} \\
\dot{x}_{4}
\end{array}\right)=\left(\begin{array}{c}
\dot{x} \\
\dot{y} \\
\ddot{x} \\
\ddot{y}
\end{array}\right)
$$

Taking into account equations (9), (10) and (12), the equation of state is:

$$
\left(\begin{array}{l}
\dot{x}_{1} \\
\dot{x}_{2} \\
\dot{x}_{3} \\
\dot{x}_{4}
\end{array}\right)=\left(\begin{array}{c}
\dot{x} \\
\dot{y} \\
\ddot{x} \\
\ddot{y}
\end{array}\right)=\left(\begin{array}{cccc}
0 & 0 & 1 & 0 \\
0 & 0 & 0 & 1 \\
-\dot{\theta}^{2} & -\ddot{\theta} & 0 & 0 \\
\ddot{\theta} & -\dot{\theta}^{2} & 0 & 0
\end{array}\right)\left(\begin{array}{l}
x_{1} \\
x_{2} \\
x_{3} \\
x_{4}
\end{array}\right)
$$




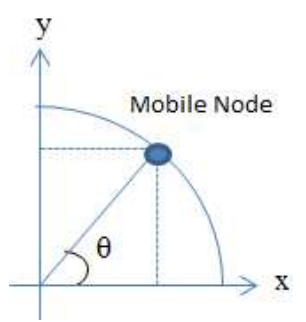

Fig. 7. position of the mobile

The signals accessible to measurement are the acceleration components, and then we take as an output.

$$
Y=h(x)=\left(\begin{array}{l}
\dot{x}_{3} \\
\dot{x}_{4}
\end{array}\right)=\left(\begin{array}{cccc}
-\dot{\theta}^{2} & -\ddot{\theta} & 0 & 0 \\
\ddot{\theta} & -\dot{\theta}^{2} & 0 & 0
\end{array}\right)\left(\begin{array}{l}
x_{1} \\
x_{2} \\
x_{3} \\
x_{4}
\end{array}\right)
$$

Finally the representation of system state is:

$$
\left\{\begin{array}{l}
\dot{X}=A(\dot{\theta}, \ddot{\theta}) X \\
Y=C(\dot{\theta}, \ddot{\theta}) X
\end{array}\right.
$$

With:

$$
\begin{gathered}
A(\dot{\theta}, \ddot{\theta})=\left(\begin{array}{cccc}
0 & 0 & 1 & 0 \\
0 & 0 & 0 & 1 \\
-\dot{\theta}^{2} & -\ddot{\theta} & 0 & 0 \\
\ddot{\theta} & -\dot{\theta}^{2} & 0 & 0
\end{array}\right) \\
C(\dot{\theta}, \ddot{\theta})=\left(\begin{array}{cccc}
-\dot{\theta}^{2} & -\ddot{\theta} & 0 & 0 \\
\ddot{\theta} & -\dot{\theta}^{2} & 0 & 0
\end{array}\right)
\end{gathered}
$$

\section{B. Luenberger observer}

Values useful in the measures are the values of $x$ and $y$ according to the (15). The difficulty is solving this equation system (non-stationary system). For this, we propose a rating system named observer luenberger [17]. The Luenberger observer takes the form:

$$
\left\{\begin{array}{l}
\dot{\hat{X}}=A \hat{X}+B(\hat{X}) u+K(Y-\hat{Y}) \\
Y=\left(\begin{array}{l}
y_{1} \\
y_{2}
\end{array}\right)=C(u) \hat{X}
\end{array}\right.
$$

The equation (18) allows the observer to ask the following:

$$
\dot{\hat{X}}=(A(\dot{\theta}, \ddot{\theta}) \hat{X}-K C(\dot{\theta}, \ddot{\theta}))(X-\hat{X})
$$

The difference between the equation (18) and Equation (19) introduced the following error:

$$
\dot{\hat{X}}=[A(\dot{\theta}, \ddot{\theta})-K C(\dot{\theta}, \ddot{\theta})] \tilde{X}
$$

With the estimation error: $\tilde{X}=X-\hat{X}$

The matrix $K$ can fix the dynamic convergence of the observer. We can consider that $F(t)=(A(\dot{\theta}, \ddot{\theta})-K C(\dot{\theta}, \ddot{\theta}))$ belongs to a convex polytope with four vertices. It suffices to find a matrix $P=P^{T}>0$ and a gain $K$ such that:

$$
P\left(A_{i}-K C_{i}\right)+\left(A_{i}-K C_{i}\right)^{T} P<0, \forall i=1,2,3,4
$$

Where $A_{i}$ and $C_{i}$ represent matrices (16) and (17) for each of the four vertices of the polytope defined by:

$\dot{\theta} \in\left[\begin{array}{ll}a_{1} & a_{2}\end{array}\right]$ et $\ddot{\theta} \in\left[\begin{array}{ll}a_{3} & a_{4}\end{array}\right]$ with known $a_{i}$.

We can solve (21) as LMI (Linear Matrix Inequality) by setting $Y=P K$ in (21). The study of eigenvalues of $(A-K C)$ is not enough because it takes a single Lyapunov matrix $P$ for the four vertices of the polytope. In order to the four LMIs in (21) have a solution; it is necessary that the unstable eigenvalues of $\mathrm{Ai}$ are observable with $C_{i}$. If we want to place the eigenvalues of $\left(A_{i}-K C_{i}\right)$ left of a vertical line " $-\alpha_{i}$ ", we replace the formula (21) by:

$P\left(A_{i}+\frac{\alpha_{i}}{2} I-K C_{i}\right)+\left(A_{i}+\frac{\alpha_{i}}{2} I-K C_{i}\right)^{T} P<0, \forall i=1,2,3,4$

\section{MAIN OBJECT OF THE ALGORITHM}

Before deployment, each sensor recognizes the successor sensor and pre-immediate successor neighboring and

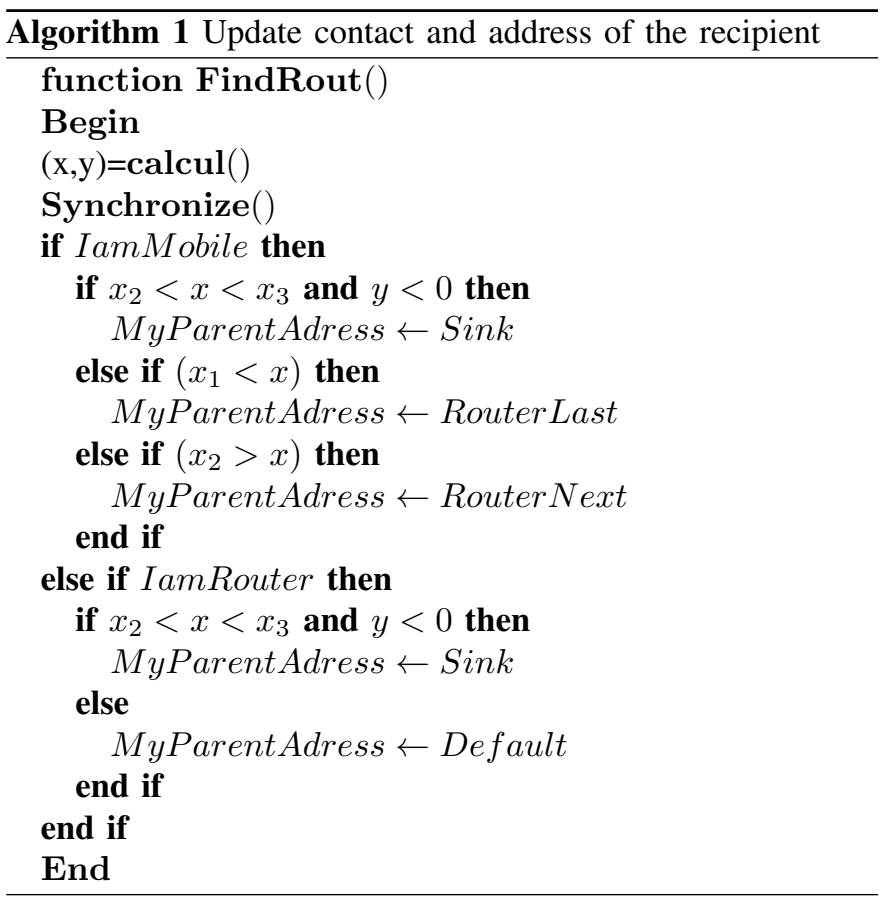

finally the routing geographical positions $P_{1}, P_{2}$ and $P_{3}$ figure5. Following deployment, each sensor measures acceleration and updates its coordinates $(x, y)$. The main idea of the algorithm is: when a mobile sensor wants to send a message (or relay a message) it compares, through his knowledge of its position, the best path (the closest geographically) between him and the destination and transmits the message to the next node in the path. The nodes are programmed with an event programming; nodes make measurements with a frequency of $100 \mathrm{~Hz}$. The function calcul return the $x$ and $y$ coordinates, the function synchronizes resets each time by the magnet,the function find rout updates the destination address according to the position of the mobile and the postions $P_{1}\left(x_{1}, y_{1}\right)$, $P_{2}\left(x_{2}, y_{2}\right)$ and $P_{3}\left(x_{3}, y_{3}\right)$, each node will have the adresses RouterLast, RouterNext and Sink. 


\section{EXPERIMENTAL PLATFORM}

To realize the approach of geographic routing, and validate the theoretical passages, we established an experimental platform described in figure8, to perform real experiments. We set the mobile nodes at the end of propellers driven by a synchronous motor; the motor is controlled by a variable speed to ensure a constant speed. We also placed a magnet to synchronize the coordinates $(x, y)$. Only one mobile node performs measures, while others perform the relay function. We implemented the same routing algorithm on all mobile nodes. The sensor nodes are used with MicaZ Mts310 senserboard, programmed with TinyOS [18] with a low power radio, and we removed the antennas to reduce the maximum focused radio. The type of accelerometer used is $A D X L 202$ with two axes $(x, y)$; the measurement range is $\pm 2 g$. The $A D X L 202$ may measure both dynamic acceleration (for example vibration), and static acceleration (for example gravity), the measured acceleration depends on the supply voltage. So, we adapted the measure to the supply voltage node.

We have been experimenting with a $20 \mathrm{tr} / \mathrm{min}$ as rotation speed; the nodes are programmed to take measurements of the acceleration with a frequency of $100 h z$ or 300 measure per rotation. We placed a sniffer node, to analyze traffic across our network (Packet loss, message flows, the information sent

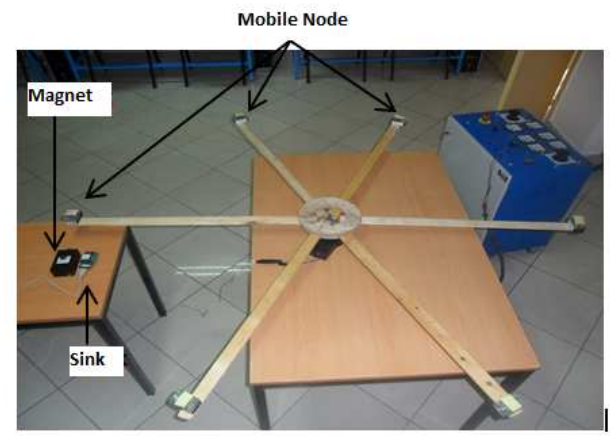

Fig. 8. Experimental Platform

by NAP, the details of association, etc.). We considered data from the senserboard Mts310 following experience with the mobile node. The movement described by the latter is uniform circular in a horizontal plane. figure 9 presents measurements of the acceleration vector from the card Mts 310 during the first sixty seconds.

\section{EXPERIMENTAL RESULTS}

The accelerometer data are biased. This is mainly due to the gravity and vibration of the propeller. Remove from the acceleration the static part is not enough. We cannot calibrate the sensor, because the static bias is not always the same value, and is different from the dynamic bias. By integrating acceleration, we get the speed, and we accumulate a bias. To avoid this pitfall we shift position once the mobile passes
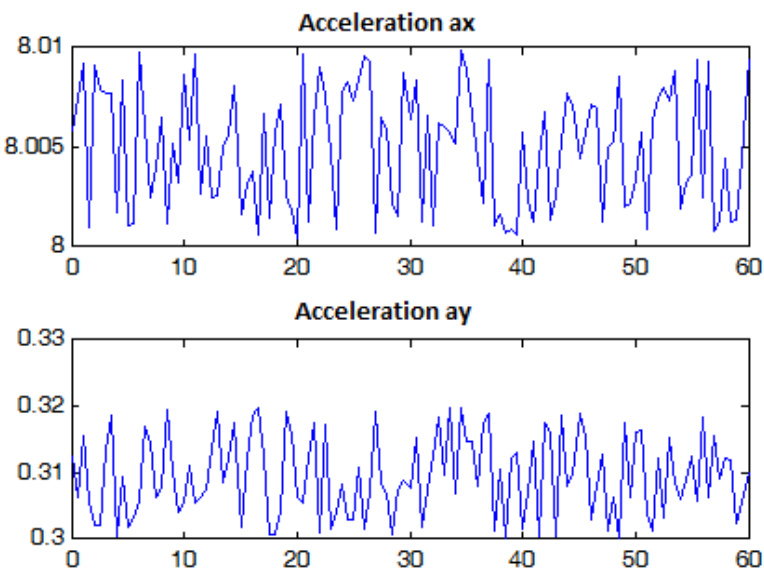

Fig. 9. Measurements of acceleration in the moving frame $\mathrm{In} \mathrm{m} / \mathrm{s} 2$ from the senserboard Mts310

through the axis $(O, \vec{X})$ it means that, the mobile set the angle to zero once it detects the presence of the magnet figure8. We can also add an observer who thwarted the drift velocity.

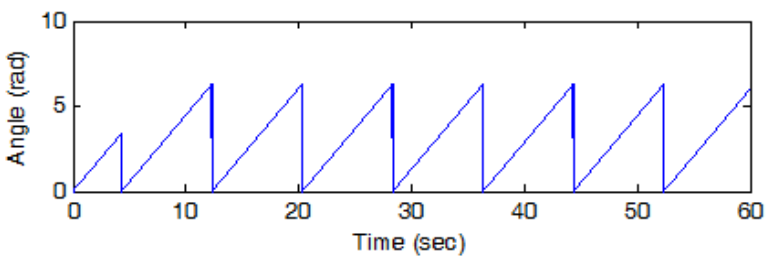

Fig. 10. the theta value calculated by the mobile in radians

At system startup, the angle theta measured gives us an idea of the angle between the mobile and the axis of the abscissa. Once the mobile node detects the presence of the magnet, it resets the theta value by zero, the theta value enables the mobile to calculate the coordinates $(x, y)$ figure8. These coordinates will permit the algorithm routing to make a decision to change the relay. During the experiment with the

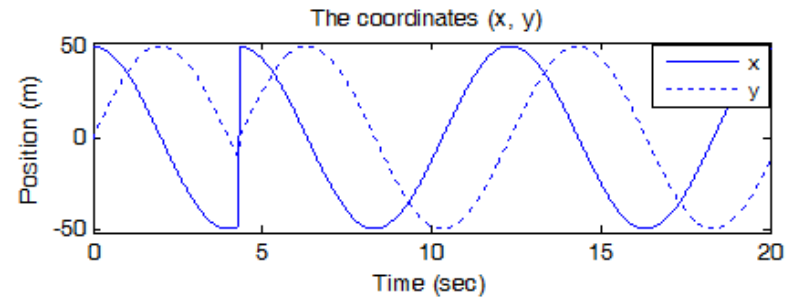

Fig. 11. Change relay

mobile node knows its position with better precision.

Figure 12 shows that the routing algorithm embedded on the mobile node changes dynamically the relay according to the position, it is attached to $R_{1}$ if its coordinate $x$ less than zero, or it attaches to the sink once its abscissa passing through the threshold $y 0$. Once the ordered $x$ becomes positive, it 


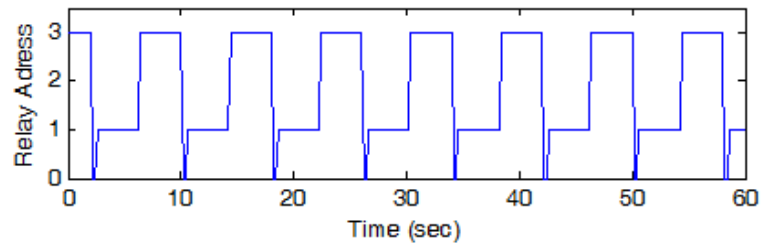

Fig. 12. Change relay

attaches to $R_{3}$. Every time the mobile detects the magnet, and initializes theta. The consumption of the mobile node is linear, these values were experimentally measured with an electronic circuit, which allows us to deduce the energy consumption, and we found the same curve with the simulation.

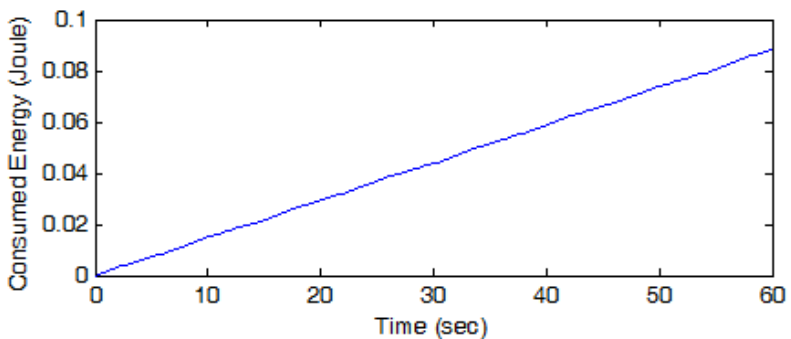

Fig. 13. delivery times of messages in seconds

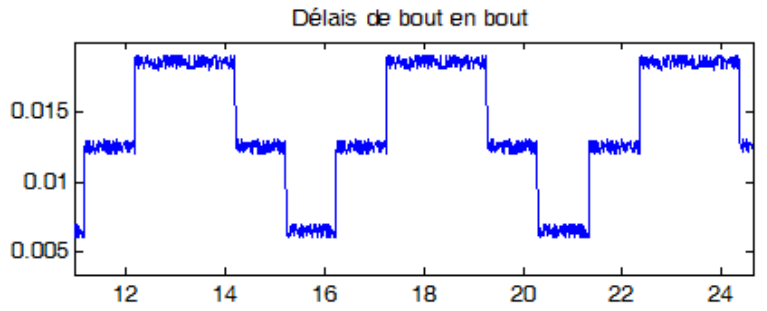

Fig. 14. Energy consumption in joules

The delay value changed figure 14 depending on the position of the mobile, and according to the number of hops. We also note that the time increases from $0.006 \mathrm{~s}$ to $0.013 \mathrm{~s}$, which explains that the mobile moves away from the sink, then the time passes its maximum value $0.019 \mathrm{~s}$ at $t=14 \mathrm{~s}$, the time drops to $0.012 s$, we can know the position of our mobile node from the delay.

\section{CONCLUSIONS AND FUTURE WORKS}

In this work we aim to optimize the routing in a mobile sensor network traveling the same path (circular path). This type of network can be used for monitoring or controlling system, it must operate under time pressure constraints in addition to optimal energy. We proposed a method for topology routing optimization of a circular path that is simple but frequently used. We also proposed a geographic routing algorithm. Experimental results have shown the contribution of this algorithm that ensures optimal delivery times. Thus it helps to improve the performance and lifetime of the network.
We plan to extend our study using directional antennas, which should provide a significant gain since the mobile knows the position of the bridge after learning. We will also develop an algorithm to adapt the radio range depending on the position and the remaining energy of nodes and a model for energy to facilitate maintenance. We also plan to extend our study to other more complex mobile trajectories.

\section{REFERENCES}

[1] K. Akkaya and M. Younis, "A survey on routing protocols for wireless sensor networks," Ad Hoc Networks, vol. 3, pp. 325-349, 2005.

[2] J. Aspnes, T. Eren, D. Goldenberg, A. Morse, W. Whiteley, Y. Yang, B. Anderson, and P. Belhumeur, "A theory of network localization," Mobile Computing, IEEE Transactions on, vol. 5, no. 12, pp. 16631678, 2006.

[3] J. Al-Karaki and A. Kamal, "Routing techniques in wireless sensor networks: a survey," vol. 11, no. 6. IEEE, 2004, pp. 6-28.

[4] J. Broch, D. A. Maltz, D. B. Johnson, Y.-C. Hu, and J. Jetcheva, "A performance comparison of multi-hop wireless ad hoc network routing protocols," in Proceedings of the 4th annual ACM/IEEE international conference on Mobile computing and networking, ser. MobiCom '98. New York, NY, USA: ACM, 1998, pp. 85-97. [Online]. Available: http://doi.acm.org/10.1145/288235.288256

[5] M. Mauve, J. Widmer, and H. Hartenstein, "A survey on position-based routing in mobile ad-hoc networks," IEEE Network, vol. 15, pp. 30-39, 2001.

[6] S. Rao, M. Pai, M. Boussedjra, and J. Mouzna, "Gpsr-1: Greedy perimeter stateless routing with lifetime for vanets," in ITS Telecommunications, 2008. ITST 2008. 8th International Conference on. IEEE, 2008, pp. 299-304.

[7] L. Junhai, Y. Danxia, X. Liu, and F. Mingyu, "A survey of multicast routing protocols for mobile ad-hoc networks," Communications Surveys \& Tutorials, IEEE, vol. 11, no. 1, pp. 78-91, 2009.

[8] I. Stojmenovic and X. Lin, "Loop-free hybrid single-path/flooding routing algorithms with guaranteed delivery for wireless networks," IEEE Transactions on Parallel and Distributed Systems (TPDS), vol. PDS-12, no. 10, pp. 1023-1032, Oct. 2001.

[9] H. Takagi and L. Kleinrock, "Optimal transmission ranges for randomly distributed packet radio terminals," IEEE Transactions on Communications, Vol. COM-32, :3, 246-257, vol. COM-32, 3, pp. 246-257, 1984.

[10] A. Cvetkovski and M. Crovella, "Hyperbolic embedding and routing for dynamic graphs," Tech. Rep., 2009.

[11] Y. Zhou, B. Gu, K. Chen, J. Chen, and H. Guan, "An range-free localization scheme for large scale underwater wireless sensor networks," Tech. Rep. 5, 2009.

[12] J. Champ and V. Boudet, "ADNL-angle: Accurate distributed node localization for wireless sensor networks with angle of arrival information," in ADHOC-NOW, ser. Lecture Notes in Computer Science, I. Nikolaidis and K. Wu, Eds., vol. 6288. Springer, 2010, pp. 177-190. [Online]. Available: http://dx.doi.org/10.1007/978-3-64214785-2

[13] H. Chu and R. Jan, "A gps-less, outdoor, self-positioning method for wireless sensor networks," Tech. Rep. 5, 2007.

[14] A. Savvides, C.-C. HAN, and M. B. STRIVASTAVA, "Dynamic finegrained localization in ad-hoc networks of sensors," in MobiCom '01: Proceedings of the 7th annual international conference on Mobile computing and networking. New York, NY, USA: ACM, 2001, pp. 166-179.

[15] F. Franceschini, M. Galetto, D. Maisano, and L. Mastrogiacomo, "A review of localization algorithms for distributed wireless sensor networks in manufacturing," International Journal of Computer Integrated Manufacturing, vol. 22, no. 7, pp. 698-716, 2009.

[16] D. Niculescu and B. R. Badrinath, "Ad hoc positioning system (aps) using aoa," in INFOCOM, 2003.

[17] D. G. Luenberger, Linear and Nonlinear Programming. Menlo Park: 2nd edition, Addison-Wesley, 1984.

[18] TinyOS, Community Forum http://www.tinyos.net. 